\title{
Community Use Skills
}

National Cancer Institute

\section{Source}

National Cancer Institute. Community Use Skills. NCI Thesaurus. Code C121190.

An assessment of the Adaptive Behavior Assessment System where community use

skills, such as shopping, getting around the community, and using community resources, are evaluated. 